\title{
HOW SAMPLING-BASED OVERDISPERSION UNDERMINES INDIA'S TIGER MONITORING ORTHODOXY
}

Arjun M. Gopalaswamy ${ }^{1}$, K. Ullas Karanth ${ }^{2,3,4}$, Mohan Delampady ${ }^{1}$, Nils Chr. Stenseth $^{5^{*}}$

1. Statistics and Mathematics Unit, Indian Statistical Institute, Bangalore Centre, Bengaluru - 560059, India.

2. Centre for Wildlife Studies, $37 / 5$, Yellappa Garden, Yellappa Chetty Layout, Sivanchetti Gardens, Bengaluru, Karnataka 560042, India.

3. Wildlife Conservation Society, Global Conservation Program, Bronx, New York 10460, USA.

4. National Centre for Biological Sciences, Tata Institute of Fundamental Research, Bengaluru - 560065, India.

5. Centre for Ecological and Evolutionary Synthesis (CEES), Department of Biosciences, University of Oslo, Blindernveien 31, NO-0316, Oslo, Norway.

- Corresponding Author: n.c.stenseth@ibv.uio.no

\section{Ackowledgements}

We thank the Nigel Yoccoz, two anonymous reviewers and the Associate Editor for helpful suggestions on the earlier version of this paper. AMG thanks the Indian Statistical Institute, Bangalore Centre and Wildlife Conservation Society, New York for funding support. We thank David W. Macdonald and Tim Coulson for encouraging us and providing administrative support to AMG during his time at Oxford. We thank Bob May, Jim Nichols, Sari C. Cunningham, Devcharan Jathanna and Varsha S. Shastry for helpful comments and assistance with the drafts. 

pressures.

\section{Abstract:}

Conservation agencies entrusted with recovery of iconic mammals may exaggerate population trends without adequate scientific evidence. Recently, such populations were termed as 'political populations' in the conservation literature. We surmise that political populations emerge when agencies are pressured to report abundances at large spatial scales for species that are difficult to survey. Indian tiger conservation agencies use an experimental approach called double-sampling using index-calibration models. A recent, mathematical, study demonstrated the unreliability of this approach in the context of India's tigers. Yet, this approach continues to be applied and even promoted by global tiger conservation agencies in other tiger range countries. In this article, we aim to: (1) discuss the ecological oddities emerging from results of India's national tiger surveys, (2) demystify the mathematics underlying the problems of this survey methodology and (3) confront these findings with results from India's recent national tiger survey of 2014 . Our analyses show that the predictions of tiger abundance using sign-based indices reported in the 2014 survey in fact vary greatly and can be severely misleading and confirming the presence of high sampling-based overdispersion and parameter covariance. We call for species conservation initiatives to implement monitoring methods that are designed to clearly answer, a priori, scientific or management objectives instead of potentially 
Main text:

\section{INTRODUCTION AND BACKGROUND}

Krebs (1991) recognized that monitoring programs must advance our

knowledge of the underlying dynamics of animal populations if they are to improve either science or conservation. Towards this end, Nichols and Williams (2006) recommend a priori designing of animal monitoring programs to answer clearly defined scientific or management questions. And in practice, Williams et al. (2002) identify two major sources of uncertainty (imperfect detection and inappropriate spatial sampling), which must be addressed while implementing monitoring programs to generate strong inferences about animal population dynamics.

Monitoring programs for some of the world's most iconic endangered mammals, however, appear to routinely ignore these profound insights leading to claims about population dynamics of such species resting on weak inferences and untested leap of faith arguments. For example, Darimont et al. (2018) explain how population trends reported by agencies for several charismatic carnivores lack adequate scientific support. Using case studies of wolves (Canis lupus) in USA and Sweden and brown bears (Ursus arctos) in

47 Romania and Canada, they demonstrate how population increases claimed by federal agencies are exaggerated. Hypothesizing that these claims serve

49 political interests, they coined the term "political populations" (Darimont et al.

502018 ) to identify such species. Therefore, it is important to assess whether

51 conservation agency claims about population trends of political populations 
52 arise from poorly framed monitoring questions, inadequate sampling designs

53 or from extraneous social considerations such as 'motivated reasoning'

54 (Kunda 1990). In this essay, we attempt to disentangle these factors based

55 on official reports of monitoring wild tigers (Panthera tigris) in India, also

56 suspected to be a political population by Darimont et al. (2018).

57

58 The tiger is an ideal 'political species' for such an investigation because of the

59 global attention and massive conservation investments it has attracted (PTI

60 2016). In this article, we first contrast official results of Indian tiger surveys

61 with ecological theory and prior scientific knowledge of tiger population

62 dynamics. Thereafter, we examine the underlying statistical factors leading to

63 the scientific inferences from these surveys. Finally, we broaden implications

64 of our results to political populations of other charismatic species.

65

66

67

68

69

70

71

72

73

74

75

\section{INDIA'S CLAIMS OF RISING TIGER NUMBERS}

The Indian country-wide official surveys of 2006, 2010 and 2014 (herein referred as "NTE surveys") report estimates of tiger population sizes at 1411 (1165-1657), 1706 (1507-1896) and 2226 (1945-2491), respectively (Jhala et al. 2011a, Jhala et al. 2011b, Jhala et al. 2015). The numbers in brackets putatively represent the range, without a clear statistical explanation of how these values are derived. Regardless, these numbers with their reported error bounds indicate significant increases in tiger numbers in India. 
76 Considering only areas that were actually surveyed (summarized from Jhala

77 et al. 2015), these numbers translate to a $17.2 \%$ increase in tiger abundance

78 and a corresponding increase of $34.4 \%$ in local tiger density, implying that

79 local tiger density $D$ rose at twice the rate of tiger abundance $N(\Delta D / \Delta N=2)$

80 between 2006-2010. Between 2010-2014, an even steeper 30\% increase in

81 tiger abundance was reported, but this time there was corresponding

82 decrease in local tiger density down to $19 \%$. These results imply a reversal of

83 the tiger population growth mechanism (from $\Delta \mathrm{D} / \Delta \mathrm{N}=2$ to $\Delta \mathrm{D} / \Delta \mathrm{N}=0.63$ )

84 after four years, with the year 2010 as the point of inflexion.

85

86 Furthermore, between 2006-2010, the surveys reported a simultaneous contraction of tiger range by $12.9 \%$ (or $11,400 \mathrm{~km}^{2}$ ). In contrast the results of the next survey interval (2010-2014) claim an abrupt reversal of the earlier pattern, reporting a range expansion of $9.4 \%$, suggesting a tiger recolonization of $7,250 \mathrm{~km}^{2}$ of new habitat (computed from Jhala et al. 2015).

These tiger population increase mechanisms imply a concave upward relationship between tiger abundance and occupancy (Figure 1) and stand in contrast to the general mechanism of a monotonically increasing, but concaving downward, relationship based on basic scientific literature (see

96 Gaston et al. 2000).

Furthermore, long-term studies of tiger population dynamics using rigorous

99 photographic capture-recapture surveys even in some better-protected tiger 100 reserves of India (Karanth et al. 2006) and Thailand (Duangchantrasiri et al. 
$1012016)$ demonstrate far lower annual rates of density increase ( 2-4\%). If

102 these survey outcomes are considered together the implication is that tiger

103 populations in large, poorly-protected, low-prey density, sink landscapes

104 exhibit higher growth rates than populations in better protected source

105 populations (Karanth et al. 2016). Therefore, the results from the Indian tiger

106 surveys stand in stark contrast to scientific understanding derived from the

107 source-sink theory in population biology (Pulliam 1988), foundational to most

108 global recovery plans for large carnivores including those for tigers in India

109 (Walston 2010, NTCA 2012).

111 Recently, Harihar et al. (2017) analyzed these NTE survey results to show

112 that increase of sampled areas in tiger 'source sites' among successive

113 surveys led to decreases in tiger density. Hence, this study (Harihar et al.

1142017 ) also contradicts implications from NTE surveys by showing that tiger

115 population sinks are indeed performing worse than reported and supporting

116 the proposals of Karanth et al. (2016) and Harihar et al. (2018), that tiger

117 population recovery rates will be far slower than expected.

119 What are the reasons for these gross ecological anomalies that arise from

120 Indian tiger surveys? The explanation by Darimont et al. (2018) is that

121 ecological claims about political populations may often be disconnected from

122 formal science. But the acceptance of the Darimont et al. (2018) explanation,

123 without critical examination, may impede our gaining an understanding of tiger

124 population biology from potential scientific serendipities (Wintle et al. 2010).

125 Therefore, here we examine the basis of India's claims on tiger numbers by 
126 assessing, in detail, the methods and models used to generate India's tiger

127 population estimates.

DOUBLE-SAMPLING USING INDEX-CALIBRATION MODELS

130 The NTE survey method was developed and implemented in 2005 as India's

131 new official tiger monitoring approach (Jhala et al. 2008) after the failure of the

132 previous 'pugmark census' method. Incidentally, the 'pugmark census' method

133 persisted for three decades until it had to be suddenly abandoned only in

1342005 due to its failure to detect the extirpation of an important tiger population

135 (Sariska; Tiger Task Force 2005), followed by the extinction of another

136 important tiger population (Panna; Special Investigation Team 2009).

138 The NTE survey method is based on the double-sampling experimental

139 approach (Eberhardt and Simmons 1987). Double-sampling was developed

140 because rigorous estimation of abundance at large spatial scales is often

141 impractical because of ecological, environmental and logistical constraints

142 (Eberhardt and Simmons 1987). When applied correctly, double-sampling

143 involves the following steps:

144 (1) Random selection of a sample of sites from a larger pool of potential sites

145 spread across a large region.

146 (2) Conduct of surveys at the sampled sites to estimate true animal

147 abundance using a rigorous, reliable, method which is typically expensive,

148 intensive and relatively difficult to implement (e.g photographic capture-

149 recapture sampling, distance sampling). 
150 (3) Conduct of a less rigorous, but practically feasible, field survey using an 151 index of animal abundance (eg. number of animal tracks $/ \mathrm{km}$ walked) at the 152 selected sites as well as across the larger region.

153 (4) Development of an 'index-calibration' model (often a simple linear

154 regression model) to establish a statistical relationship between true animal 155 abundance (2) and its putative index (3).

156 (5) Estimation of animal abundance for the larger area using the index157 calibration model (4).

158 Consequently, the reliability of results from the double-sampling approach will 159 rest on the performance of the index-calibration model (step 4 from above) 160 developed.

162 In the past, practical index-calibration experiments have yielded divergent 163 results in terms of efficiency (see Gopalaswamy et al. 2015a,b and citations 164 therein). Gopalaswamy et al. (2015a,b) mathematically modeled typical index165 calibration models to analyze key contributors that produce such divergent 166 outcomes in real world field surveys. In Appendix 1, without getting into all the 167 mathematical complexities of Gopalaswamy et al. (2015a,b) we, heuristically, 168 summarize the concepts of sampling-based overdispersion (SOD) and 169 parameter covariation.

175 Based on mathematical and simulation-based explanations presented in

176 Appendix 1, we reassess the inferences of empirical, large-scale, tiger 
177 surveys discussed in Gopalaswamy et al. (2015a,b). We elaborate on certain

178 empirical details that were implicitly assumed in Gopalaswamy et al. (2015a,b)

179 to contextualize the concepts for conservationists.

\section{(i) Assessing the predictive strengths of tiger sign index-calibration}

182 experiments

183 Gopalaswamy et al. (2015a,b) examined two different tiger sign index-

184 calibration experiments (briefly labeled IC1 and IC2), which produced

185 extremely divergent calibration successes. As a framework for statistical

186 comparisons, they assumed that over India's tiger occupied habitat of about

$187 \sim 80000 \mathrm{~km}^{2}$ (Jhala et al. 2011a), there could be a potential pool of more than

188400 sites each of $\sim 200 \mathrm{~km}^{2}$ size (approximately the size of sites used in the

189 two experiments). In the two experiments, at each site, an estimate of tiger

190 density was derived from photographic capture-recapture sampling (Karanth \&

191 Nichols 1998) from replicated surveys (see Karanth et al. 2004, Jhala et al.

192 2011b, for field work details).

194 At these sampled sites, tiger signs (scats and tracks) were counted by

195 observers walking along trails to derive encounter rate indices (number of

196 scats or track sets $/ \mathrm{km}$ walked). These index count data, $S \mid N$ were fitted to

197 linear regression models by Ordinary Least Square (OLS) solutions. The first

198 experiment (IC1), with a sample size of 21 sites, returned a high $R^{2}$ estimate

199 of 0.95 (as reported in Jhala et al. 2011a), whereas the second experiment

200 (IC2), with a sample size of 8 sites, returned a low $R^{2}$ estimate of 0.0004 (as 
201 computed using the Im function in R that uses the Eqn 4 from Kvalseth 1985

202 for estimating $R^{2}$ ).

203 We note that the slope of these index-calibration relationships is $\beta=k p^{*}$, where

$204 p^{*}$ is the average detection probability per individual. Because the index

205 based on tiger signs was computed from counts obtained in single sweep of

206 each site, we set the value of $k=1$. Thus, making $\beta=p^{*}$ in this case. If we apply

207 the mathematical formula derived by Gopalaswamy et al. $(2015 a, b)$ for

208 population $R^{2}$ to the binomial model (less overdispersed case) we can obtain

209 the estimate of detection probability $p^{*}$. This computed value is seen to be

210 high for IC1 $\left(\hat{p}_{I C 1}^{*}=0.92\right)$ and low for IC2 $\left(\hat{p}_{I C 2}^{*}=0.0003\right)$. These two slopes

211 are plotted as blue lines in Figure 4.

212

(ii) Estimating the true value of $p^{*}$ for tiger sign index-calibration experiments

215 From the larger tiger distribution surveys conducted by Jhala et al. (2011a)

216 and Karanth et al. (2011), the average $p^{*}$ from these two surveys has an

217 estimated value of 0.125 (represented by the red line in Figure 4), lying

218 between the two blue lines.

220 This result clearly shows that sample sizes in both experiments (IC1=21 sites

221 and IC2=8 sites) were far too small to accurately reflect the population

222 characteristics. Secondly, the sampled sites selected non-randomly were not

223 truly representative of the assumed larger pool of $>400$ sites because they

224 both failed converge on the correct population estimate of average $p^{*}$. This

225 implies that both these index-calibration models have poor predictive power 
226 across the wider spatial region of interest. Furthermore, in IC 1 , the 21 sites

227 selectively excluded southwestern Indian region (see Jhala et al. 2011a,b). As

228 seen earlier, the presence of large SOD makes index-calibration models very

229 data-hungry, and any such selective, and potentially biased, sub-sampling of

230 sites will compound the predictive inefficiency of these models.

231

232

(iii) Factors likely to influence the potentially large variation in $p^{*}$

233 We note that the tiger sign index-calibration models assume $k$ is a constant

234 and is equal to 1 . In reality, $k$ will be a function of the number of index values

235 accumulated during the days prior to sampling and hence the constant $k$

236 assumption may itself be unreal. For example, in drier forests (that cover

237 about $50 \%$ of tiger habitats in India), tiger scats may remain intact for days

238 prior to the counting, whereas they disappear rapidly in wetter regions.

239 Although, Gopalaswamy et al. (2015a,b) did not derive explicit expressions for

240 such variations in $k$, by assuming $k$ is a constant, such an assumption will

241 further, unrealistically, imply that the slope of $\beta$ is entirely due to the variation

242 in $p^{*}$. We also note that $p^{*}=\alpha p$. In this context, $p$ refers to the detection

243 probability of an individual tiger and its magnitude being determined primarily

244 by the type of substrate (see Figure 3). Similarly, $\alpha$ represents the total

245 fraction trails sampled. For example, in a photographic capture-recapture

246 study, Karanth et al. (2004) demonstrated that detection probability $p^{*}$ was

247 much higher for tigers in the denser forests of Tadoba (0.174) and Bhadra

248 (0.22), compared to open forests at Panna (0.039) or Bandipur (0.055) sites.

249 This was possibly because the unknown proportion of trails used by tigers that

250 were actually sampled, $\alpha$, is relatively higher in denser forests with lower 
251 density of trails. We note here though that the value of $p$ can potentially be

252 very high only in exceptional circumstances, for example, detecting tiger

253 tracks in snow in Russia (Miquelle et al. 2015) but $\alpha$ will continue to be

254 dictated by the relative sampling effort per unit area.

256 We also note that in both the above experiments IC1 and IC2, no particular

257 spatial sampling design was employed to select trails. This factor also would

258 additionally to contribute to causing biases in the estimate of $\alpha$. Therefore,

259 overall, the combined uncertainties of $k, \alpha$ and $p$ are likely to contribute to the

260 large variation seen in the value of $p^{*}$, resulting in the high overdispersion

261 observed in such survey data.

\section{SAMPLING-BASED OVERDISPERSION IN INDIA'S TIGER SURVEY OF}

\section{$\underline{2014}$}

The resulting implication is that if SOD is not taken into account then any

267 estimate of tiger abundance at the national scale will be non-robust and

268 potentially flawed. To assess the generality of this conclusion, we evaluate

269 estimates of tiger abundance from the previously unexamined NTE survey of

2702014 (Jhala et al. 2015).

272 The calibration models developed during this survey comprised of a few

273 environmental covariates in addition to tiger signs as explanatory variables to

274 model tiger density. By the measure of relative importance of covariates (see

275 Burnham and Anderson 2002), the survey results confirm that tiger sign index 
276 is the most important predictor of tiger density as this covariate is featured in

277 the three main landscape models. This shouldn't be surprising because signs

278 of tigers are due to the presence of tigers. Whether treating tiger sign index as

279 a relevant covariate in such modeling efforts is a separate question worthy of

280 independent investigation. What is relevant to us here is whether there is SOD

281 present in the relationship between tiger signs and tiger abundance.

283 In all the intensively monitored sites, the surveys estimated the beta

284 coefficients corresponding to the tiger sign index covariate to be

$\operatorname{bet}_{S G}\left(\operatorname{SE}\left(\right.\right.$ beta $\left.\left._{S G}\right)\right)=0.1(0.06)$, beta $_{C I E G}\left(S \hat{E}\left(\right.\right.$ beta $\left.\left._{C I E G}\right)\right)=0.258(0.028)$ and

bet $_{W G}\left(S \hat{E}\left(\right.\right.$ bet $\left.\left._{W G}\right)\right)=1.01(0.08)$, where SG, CIEG and WG correspond to

abbreviated forms of Shivalik-Gangetic Plains, Central-Indian and Eastern

288 Ghats and Western Ghats, respectively. We note here that the definition of

289 beta in these reports will differ from our definition of $\beta$ earlier in that beta is

290 meant to represent the rate of change of animal density for a unit increase in

291 the signs detected. However, our purpose is to investigate SOD and

292 parameter covariation and these estimates of beta serve that purpose well

293 enough.

295 The full mathematical specification of the model used in Jhala et al. (2015) is

296 not available. However, from the model coefficients reported, they appear to

297 be generated using the default log-linear model in the package secr (Efford

298 2019). Therefore, the above beta estimates must be back-transformed

299 exponentially for appropriate interpretation. Accordingly, one unit increase in 
300 the tiger sign index results in a corresponding exponential increase in tiger

301 density of (i) $10.5 \%$ in the Shivalik-Gangetic Plains (ii) $29.4 \%$ in the Central

302 Indian and Eastern Ghats landscape and (iii) a massive $174.6 \%$ increase in

303 tiger density in the Western Ghats. Such a massive variation in the influence

304 of tiger sign index on tiger density demonstrates the presence of enormous

305 SOD inherent in the population. Interestingly, these estimates of beta indicate

306 that the non-linear nature of the relationship between tiger sign index and tiger

307 density is very pronounced, perhaps indicating a strong interaction between $p$

308 and/or $\alpha$ and $N$ as discussed earlier.

310 Such variations arising from SOD mean that when these beta estimates are

311 utilized to estimate tiger abundance over wider regions (eg: at regional and

312 national levels) or used to assess changes in tiger abundance over time, the

313 resulting trends can essentially lack any real ecological meaning. And the

314 large variation in the extent of non-linearity further weakens predictions of

315 tiger abundance at regional and national levels.

\section{CONSERVATION IMPLICATIONS}

319 The beta estimates reported in Jhala et al. (2015) strongly confirm the

320 presence of a high degree of SOD and parameter covariance in tiger index-

321 calibration experiments used in Indian tiger surveys. The temporal variations

322 in the beta estimates and the unpredictable changes in the form of the index-

323 calibration relationship itself make the prediction of animal abundance at large

324 spatial scales very unreliable. We conclude that changes in the tiger 
325 population size and occupancy reported from Indian tiger surveys, which are

326 so anomalous in the context of ecological rationale (see Introduction), are

327 outcomes from an unreliable method used which spuriously seems to

328 challenge existing understanding of animal population dynamics in ecology.

330 There are several management implications that arise from our analyses. It is

331 essential that the raw data from the past NTE surveys (Jhala et al. 2008,

332 Jhala et al. 2011a, Jhala et al. 2015) be thoroughly re-analyzed to account for

333 the hitherto ignored underlying SOD and parameter covariance we have we

334 have uncovered here. Only such a re-analysis can correct these tiger

335 population estimates, by fully recognizing the true underlying uncertainties

336 that have been previously ignored. We note that, analytically, the SOD

337 problem can at least be reduced partially by accounting for spatial random

338 effects (Dey et al. 2017). More importantly, the understanding of the effects of

339 SOD provides an opportunity for conservation agencies to introspect deeply

340 about properly designing monitoring programs keeping in view suggestions of

341 Nichols and Williams (2006) given the enormous resources (manpower, time

342 and money) spent on Indian tiger surveys as detailed in Jhala et al. (2015).

344 CONSERVATION OUTCOMES: DOES SCIENCE INFLUENCE POLICY?

345 Accepting the premise that species conservation programs should be based 346 on science and evidence, the conservation implications discussed above can

347 serve as a template for future tiger monitoring policies. But, as discussed in

348 Darimont et al. (2018), this often will not be the case with political populations. 
350 Our understanding of the presence of large SOD in India's official tiger

351 estimation approach was based on the 2010 survey results and presented in

352 Gopalaswamy et al. (2015a). Coincidentally, this publication appeared only a

353 month after India announced that its tiger population had risen by $30 \%$ during

354 the years 2010-2014.

355

356 Surprisingly, instead of seeking more information, studying implications of

357 Gopalaswamy et al. (2015a) in detail, or by engaging in a formal scientific

358 debate, some scientists and officials associated with the NTE survey, rushed

359 to the journal demanding, summarily, the retraction of Gopalaswamy et al.

360 (2015a) (Vishnoi 2015, Kempf 2016). Given that our analysis of the NTE

361 survey of 2014 discussed here further strengthens arguments advanced by

362 Gopalaswamy et al. (2015a,b), this conservation outcome of demanding

363 retraction is perplexing.

365 We note with some concern that in spite of the presence of a body of scientific

366 evidences pointing towards major concerns about India's tiger population rise

367 claims (Gopalaswamy et al. 2015a,b, Karanth et al. 2016, Harihar et al. 2017,

368 Harihar et al. 2018) major international conservation agencies, such as the

369 Global Tiger Forum (GTF), Global Tiger Initiative (GTI), and the World Wide

370 Fund for Nature (WWF), continued to endorse claims of success made by

371 Indian Tiger Surveys (WWF 2016). Consequently, India's tiger conservation

372 budget jumped from USD $\$ 70$ million to $\$ 144$ million in 2016 to reward this

373 achievement (PTI 2016). 
375 Of even greater concern is the fact that index-based monitoring methods continue to be uncritically employed by India's National Tiger Conservation

377 Authority and Wildlife Institute of India (Jhala et al. 2017) and even being

378 further promoted by GTF and by the GTI in other tiger range countries that are

379 just initiating national monitoring programs (see Dey et al. 2015 for an

380 example of from Bangladesh). Similarly, Nepal also claimed that their tiger

381 numbers doubled in a relatively short period of time (Davis 2018) without

382 adequate scientific support.

384 More recently, Qureshi et al. (2018) archived in a public preprint repository a

385 critique of Gopalaswamy et al. (2015a,b), but at the same time supporting 386 claims of the NTE survey of 2014 (Jhala et al. 2015). Since we demonstrate 387 that Jhala et al. (2015) only buttresses the scientific findings of Gopalaswamy 388 et al. (2015a,b), especially with regards to SOD and parameter covariance, 389 we find that this critique (Qureshi et al. 2018) too appears to be a paradoxical 390 conservation outcome (Gopalaswamy 2019), and contrary to science-based 391 implications.

\section{DISCUSSION}

395 As we elucidate in this article, the combined phenomena of sampling-based 396 overdispersion and parameter covariance can induce a large amounts of 397 uncertainty in predictions of animal abundance over large spatial and 398 temporal scales. This implies that the claims of a $58 \%$ tiger population rise in 399 India over the past 8 years (from 2006-2014) based of estimates from the 
400 three NTE surveys (Jhala et al. 2008, Jhala et al. 2011a, Jhala et al. 2015)

401 lack reliable scientific support.

402

403 Our analysis of these additional sources of uncertainty, however, helps in 404 explaining the ecological paradoxes (see Introduction) that the survey results 405 lead to. For example, the unusual concave upwards relationship between tiger occupancy and abundance (Figure 1; derived from the summary table in Jhala

407 et al. 2015), the reversal of the source-sink mechanism (Karanth et al. 2016, 408 Harihar et al. 2017) and the unusually the high estimates of tiger population 409 growth rates in source populations (Karanth et al 2006, Duangchantrasiri et al. 410 2016) all appear to be consequences of inferential problems arising from 411 sampling-based overdispersion and parameter covariance.

413 In general, we believe that such misreading of population growth patterns

414 resulting from inferences based on vague survey methodologies can be

415 detrimental to wildlife conservation. Just over a decade ago a similar 416 disregard to scientific findings, using an earlier flawed survey methodology

417 known as the pugmark census (Karanth et al. 2003) had hidden real tiger

418 population collapses in India. At that time, India's tiger numbers were reported

419 to have reached 3642 individuals (Ramesh 2008). This was followed by

420 abandonment of the census method, as tiger populations collapsed from a

421 wave of poaching in two key reserves in India (Tiger Task Force 2005,

422 Chundawat 2018). 
424 The current claim of an upward trend in tiger numbers is reminiscent of that

425 previous conservation 'bubble'. Based on our results and more general

426 suggestions of (Nichols and Williams 2006), we question the very purpose of

427 conducting such massive, resource intensive surveys across large regions

428 without first addressing challenge of SOD and parameter covariance, which

429 remains challenging if not intractable.

431 We note that at the high profile Global Tiger Summit in St. Petersburg in 2010, 432 doubling the global number of wild tigers by 2022 was proclaimed as the goal 433 and financial commitments of about US $\$ 330$ million were pledged (Watts 434 2010). We worry that large financial commitments in species conservation 435 initiatives may subconsciously create social pressures on conservation 436 agencies leading to motivation or cognitive bias (Kunda 1990, Kahneman 437 2013) influencing either the survey design itself or the reporting of results from 438 surveys. For example, by claiming rising tiger numbers, Indian conservation 439 agencies obtained an immediate increase in their tiger conservation budgets 440 (PTI 2016). And when non-robust monitoring survey methodologies (eg: with

441 inherently large SOD and/or parameter covariance) are employed, they can

442 even dangerously legitimize any popular claim about population trends

443 because such estimates can understate the true, but much wider, confidence 444 intervals. We observe the similarity of this situation to the 4-way categorization 445 by Pielke (2007) corresponding to the category of "high scientific uncertainty, 446 popular choice". 
448 We therefore stress the importance of structuring a sound monitoring program

449 in species conservation initiatives (Nichols and Williams 2006, Karanth and

450 Nichols 2017). While claims about population increases or decreases may

451 meet the goal of increasing public support and assist in raising more funds for

452 conservation, we argue that this line of reasoning can be hugely detrimental to

453 species conservation. First, such an approach will tend to benefit the most

454 advertised conservation strategy as opposed to the most effective one.

455 Consequently, those invested in solving on-ground conservation or scientific

456 problems could potentially be pressured into investing time and effort in

457 marketing and outreach. Second, monitoring programs that do not truly

458 advance scientific knowledge will undermine the entire discipline of field

459 ecology itself. For example, with respect to our above example with tigers,

460 the unexplainable concave upward relationship of tiger occupancy-abundance

461 dynamics (Figure 1) or the inexplicable accelerated growth rate of tiger

462 populations only raises more unsolvable ecological questions rather than

463 providing good answers.

465 We argue that when conservationists fail to keep pace with novel scientific

466 methodologies, any claimed estimate will prima facie be non-robust. If

467 massive changes in tiger numbers is attributed to change in methods (eg:

468 drop in India's tiger numbers from 3642 tigers (Ramesh 2008) to 1411 tigers

469 in 2006 (Jhala et al. 2008)) or due to unreliable methods (as analyzed here) it

470 has little value to either science or conservation because monitoring can no

471 longer provide useful information to conservationists in real time. 
473 Our article attempts to convey and contextualize the mathematical and

474 empirical findings of Gopalaswamy et al. (2015a,b) to conservationists,

475 especially in the context of India's claims of tiger population rise. But there is

476 vast scope for further research on this theme. If we ignore the presence of

477 SOD and parameter covariation for the moment, it is ecologically interesting to

478 assess these tiger population dynamics in the context of biological

479 overdispersion (May 1978). Since Jhala et al. (2015) and Gopalaswamy et al.

$480(2015 a, b)$ demonstrate the difficulty of applying double-sampling in practice

481 for tigers, it becomes relevant to derive the parameters for the population level

482 overdispersion caused by variation in detection rates, even though the

483 relevance of using indices for regional population level estimation may remain

484 futile (see Belant et al. 2019, in the case of lions in Serengeti).

485

486 With respect to tigers, we recommend that monitoring investments be targeted

487 to reliably understand the drivers influencing vital rates (survival, recruitment

488 and movement) at critical tiger source populations (Karanth et al. 2006,

489 Duangchantrasiri et al. 2016, Walston et al. 2010). Complementarily, we

490 recommend landscape scale, sign surveys (conducted once in 4-5 years) to

491 track and understand the mechanisms of tiger range contractions, expansions

492 and connectivity (Karanth and Nichols 2017).

494 More generally, we propose that animal monitoring programs, particularly of

495 political populations such as tigers, be designed and implemented entirely to

496 answer sound scientific and management objectives (Krebs 1991, Nichols and

497 Williams 2006) rather than be influenced by social or fund raising pressures 
498 from extraneous sources. Such a focus will help prevent charismatic large

499 carnivore populations to avoid the risk of being stigmatized as a political

500 population (Darimont et al. 2018).

501

502

503

504

505

506

507

508

509

510

511

512

513

514

515

516

517

518

519

520

521

522 


\section{References}

524

Belant et al. (2019). Track surveys do not provide accurate or precise estimates of lion density estimates in Serengeti. Global Ecology and Conservation: e00651.

528 Ed). New York: Springer-Verlag. Years of Research in Panna National Park. New Delhi: Speaking Tiger Publishing Pvt. Ltd.

Darimont, C. T. et al. (2018). Political populations of large carnivores. Conservation Biology 32: 747-749. population. Available: https://www.worldwildlife.org/pressreleases/nepal-set-to-become-first-country-to-double-wild-tigerpopulation 

abundance at large spatial scales using data from multiple sources. JABES 22, 111-139. population in Southeast Asia in the context of improved law enforcement. Conserv. Biol. 30: 639-648. Applied Ecology: 37, 39-59. experiments: counting tigers at macroecological scales. Methods Ecol Evol. 6: 1055-1066. 6: 1067-1068. 
Gopalaswamy, A. M. (2019). REVIEW.

Harihar, A. et al. (2017). Defensible inference: questioning global trends in tiger populations. Conservation Letters 10: 502-505. range. PLOS One 13(11): e0207114.

583 prey in India, 2006. National Tiger Conservation Authority, Government of India, New Delhi and Wildlife Institute of India, Dehra Dun, TR2008/001. India, New Delhi and Wildlife Institute of India, Dehra Dun, TR2011/003. Jhala, Y. V. et al. (2011b). Can the abundance of tigers be assessed from their signs?. Journal of App Ecol: 48, 14-24. Jhala, Y. V. et al. (eds) (2011a). Status of tigers, co-predators and prey in India, 2010. National Tiger Conservation Authority, Government of prey in India, 2014. National Tiger Conservation Authority, New Delhi and Wildlife Institute of India, Dehra Dun, TR2015/021. 

tigers, co-predators, prey and their habitats. Fourth edition. Technical Publication of National Tiger Conservation Authority, New Delhi and the Wildlife Institute of India, Dehradun, TR-2017/012.

606 141-146. landscape scale: occupancy modeling of tigers from sign surveys. 
prey populations. Singapore: Springer.

Karanth, K. U. et al. (2016). Statement of concern by Tiger Biologists.

http://newsroom.wcs.org/News-

$\underline{\text { Releases/articleType/ArticleView/articleld/8872/Statement-of-Concern- }}$ by-Tiger-Biologists.aspx. Viewed on 1 September 2016.

635

Kempf, E. (2016). Far from recovering tigers may be in worst decline in a Century. www.newscientist.com/article/2090507-far-fromrecovering-tigers-may-be-in-worst-decline-in-a-century/. Viewed on 1 September 2016. population dynamic studies. Ibis: 133, 3-8. Bulletin: 108, 480-498. phenomenological model. Journal of Animal Ecology 47, 833-844. 
Nichols, J. D. \& Williams, B. K. (2006). Monitoring for Conservation. TREE: $21,668-673$.

656 NTCA (2012). A Protocol on Phase-IV Monitoring. Technical Document No. $1 / 2011$.

659 PTI (2016). India now has $70 \%$ of the world's tiger population. http://indianexpress.com/article/india/india-news-india/tiger-populationin-india-prakash-javadekar-narendra-modi/. Viewed on 1 September 2016.

664 policy. Cambridge University Press. American Naturalist: 132, 652-661.

670 case of inappropriate data and deficient science. PeerJ Preprints 6:e27349v1 https://doi.org/10.7287/peeri.preprints.27349v1 

from Panna Tiger Reserve. of Environment and Forests, Government of India. questioning tiger population.

http://articles.economictimes.indiatimes.com/2015-04-

\section{$\underline{23 / n e w s / 614576871 \text { tiger-census-national-tiger-conservation- }}$} authority-research-paper. Viewed on 18 May 2016.

695 Walston, J et al. (2010). Bringing the tiger back from extinction - a six percent solution. Plos Biology lingering doubts. https://www.theguardian.com/environment/2010/nov/24/tiger-summit$\underline{\text { vladimir-putin }}$ 

Populations: Modeling, analysis and decision making. San Diego, California: Academic Press.

709 unknown unknowns. Ecology Letters: 13, 1325-1337.

712 
Figure legends:

738 Figure 1. The occupancy-abundance relationship of India's tigers. The concave upward relationship apparently challenges the existing monotonically-increasing, concave downward, occupancy-abundance relationship discussed in ecological literature.

743 Figure 2. Illustration of the contrasting estimates of $p^{*}$ under the binomial 744 model of tiger index-calibration, $N$ versus $S$ (which is conditional on $N$ ). The

745 lines are generated by the model $S \mid N \sim \operatorname{Binomial}\left(k N, p^{*}\right)$, so that $\mathrm{E}(S \mid N)=k N p^{*}$.

746 The sampling occasion $k$ is assumed to be a constant with a value of one. The

747 two blue lines represent sample $p^{*}$ estimates from two different tiger index-

748 calibration experiments. The red line represents the line generated by a $p^{*}$

749 estimate from an independent survey of the larger population of sites over

$750 \quad$ Indian landscapes.

751

752 Figure 3: Photo-trapped images of tigers on contrasting substrate types. A

753 dusty substrate (top) is conducive for detecting tiger tracks yielding a high

754 detection probability $p$. In contrast, it is virtually impossible to detect tracks of 
755 tigers on leaf-littered, grassy, substrate types (bottom) yielding a low detection

756 probability $p$. Picture courtesy of Ullas Karanth/WCS.

757

758

759

760

761

762 Figures:

763

$764 \quad$ Figure 1)

\section{Comparison of Tiger Abundance vs Occupancy}

(For years 2006, 2010 and 2014 respectively)

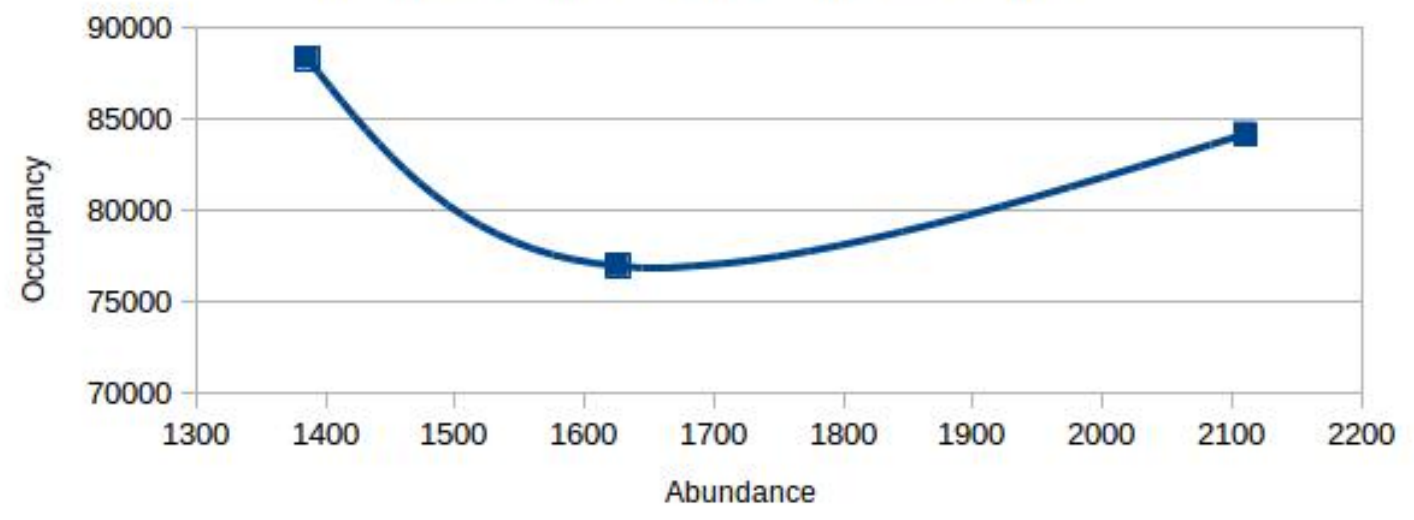

765

- - Years $(2006,2010,2014)$

766

767

768

769

770

771 
Varying slopes in tiger index-calibration experiments

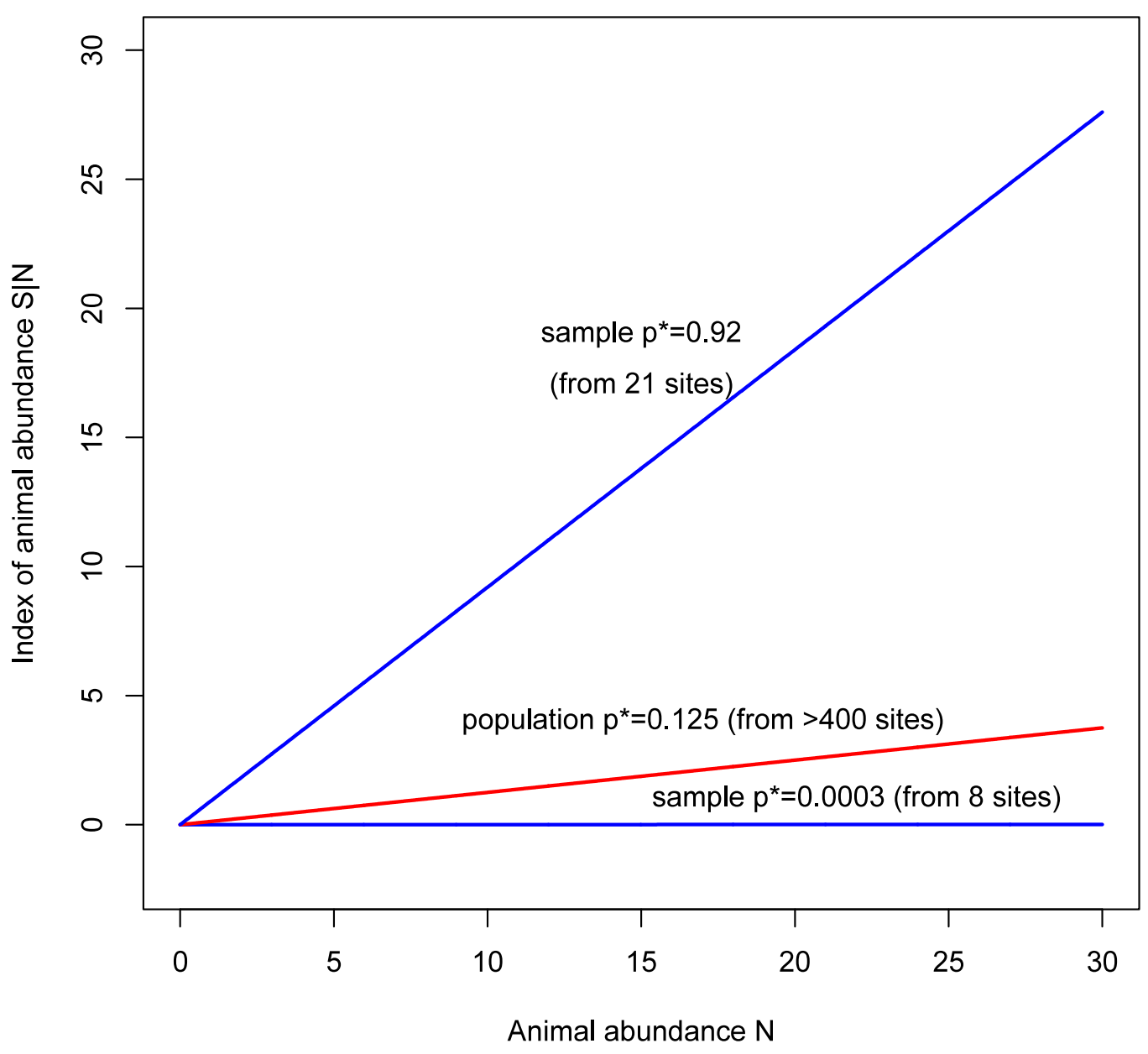


bioRxiv preprint doi: https://doi.org/10.1101/708628; this version posted July 19,2019 . The copyright holder for this preprint (which was not certified by peer review) is the author/funder. All rights reserved. No reuse allowed without permission.

\section{li}

783

784

785

786

787

788

789

790

791

792

793

794

795

796

797

798

799

800

801

802

803

804

805

806

807 


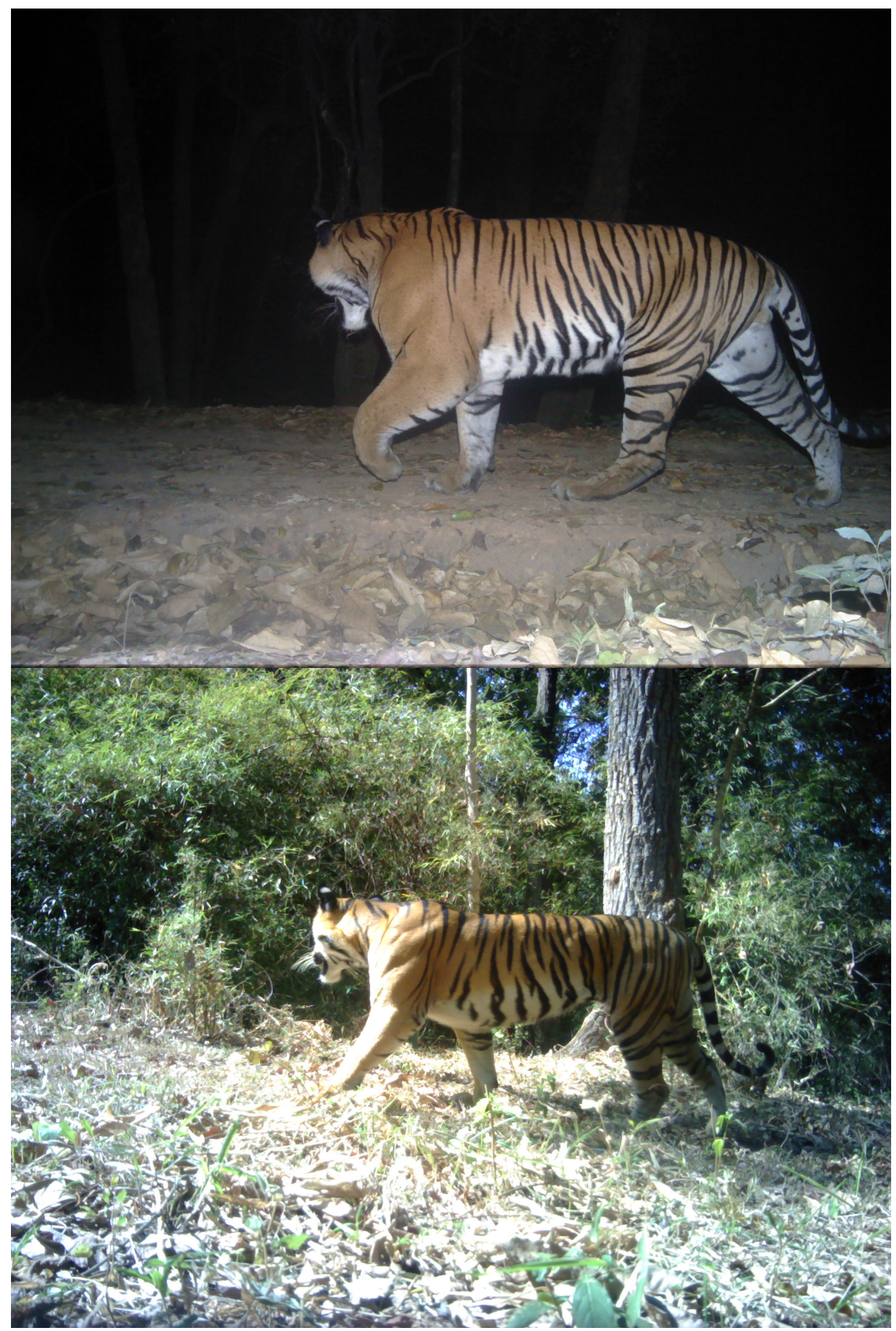


bioRxiv preprint doi: https://doi.org/10.1101/708628; this version posted July 19,2019 . The copyright holder for this preprint (which was

not certified by peer review) is the author/funder. All rights reserved. No reuse allowed without permission.

\section{li}

812

813

814

815

816

817

818

819

820

821

822

823

824

825 
SUPPLEMENTAL INFORMATION

APPENDIX 1) INDEX CALIBRATION MODELS: Summary of concepts from Gopalaswamy et al. (2015a,b)

\section{Basic Statistical Concepts}

$\underline{\text { The binomial and beta-binomial index-calibration models }}$

As in Gopalaswamy et al. (2015a,b), we define $\mathrm{N}$ as the true animal abundance at a site and $\mathrm{S}$ as the corresponding index of abundance measured at the site. We assume $\mathrm{N}$ is known noting that Gopalaswamy et al. $(2015 a, b)$ did not require this simplifying assumption. We assume that a selected site is sampled over k occasions independently. If $p^{*}$ is the probability of detecting an individual animal at this site during one occasion, then the index over $\mathrm{k}$ occasions is modeled as $\mathrm{S} \mid \mathrm{N}, \mathrm{k}, \mathrm{p}^{*} \sim$ Binomial $\left(k N, p^{*}\right) . S \mid N, k, p^{*}$ (or $S \mid N$, for simplicity) is read as $S$ conditional on $\mathrm{N}, \mathrm{k}$ and $\mathrm{p}^{*}$. This binomial model has the expected value (or expectation) $E(S \mid N)=k N p *$. For example, if we conduct a survey of animals at a single site on 3 occasions, with the true abundance being 20 animals, and the detection probability per occasion being 0.1 , then the average count from such a survey is $\mathrm{kNp}^{*}=(3)(20)(0.1)$, or 6 encounters. For a large number of repeated experiments of this kind, the experiment-toexperiment variation in counts at this site (with $\mathrm{N}=20$ ) is described by $\operatorname{Var}(\mathrm{S} \mid \mathrm{N})=k N p^{*}\left(1-\mathrm{p}^{*}\right)=6(1-0.1)=5.4$

In the above example, we have assumed detection probability $\mathrm{p}^{*}$ to be unvarying. In reality, there are many sources that can induce variation in $\mathrm{p}^{*}$ either temporarily or spatially. To account for such variation, 
26 Gopalaswamy et al. (2015a,b) developed a beta-binomial version of the

27 model, so that $\mathrm{S} \mid \mathrm{N}, \mathrm{k}, \mathrm{a}, \mathrm{b} \sim$ Beta-binomial(kN, a, b). Here, the detection

28 probability is described as a random quantity drawn from a beta

29 distribution with shape parameters a and b. Under such a model, the

30 variation in the animal counts obtained will always be larger than in the

31 binomial case (implying that $\operatorname{Var}(\mathrm{S} \mid \mathrm{N})>5.4$ if there is variation in $\mathrm{p}^{*}$ ). In

32 either case, the variance of the count increases when $\mathrm{N}$ increases. This

33 critical phenomenon is defined statistically as overdispersion. We

34 specifically define this phenomenon as 'sampling-based overdispersion

35 (SOD)' to distinguish it from 'biological overdispersion' traditionally defined

36 in ecology to represent the heterogeneity in abundances over space (May 37 1978).

(ii) Slopes of the index-calibration experiments

A good index-calibration experiment involves conduct of similar surveys at

42 index, $\mathrm{S} \mid \mathrm{N}$, from all these sites. For a fixed value of $\mathrm{k}$ and mean $\mathrm{p}^{*}$, the average slope, say $\beta$, of this relationship is given by $\mathrm{kp}^{*}$ for the binomial

45 defined earlier, for example applying a coefficient of variation in $p^{*}$ of 0.4

46 for the beta-binomial case, we can simulate multiple data points for two

47 imaginary index-calibration experiments (see Figures 1a,1b). The

48 divergent, flash-light like, spread of these data points (in blue circles)

49 indicates the extent of SOD inherent in such data. 
$51 \quad$ (iii) Measuring predictive strength in index-calibration experiments

52 The strength of an index-calibration experiment can be assessed either

53 from graphical visual assessments or using formal statistical measures.

54 Gopalaswamy et al. $(2015 a, b)$ used the coefficient of determination $\mathrm{R}^{2}(\mathrm{a}$

55 goodness-of-fit measure commonly used in the applied sciences) to

56 assess predictive strengths index calibrations under both the binomial

$57 \quad$ model and beta-binomial models. They showed that strong predictive

58 relationships $\left(R^{2}\right.$ is close to 1$)$ are obtained when detection probability $\mathrm{p}^{*}$

59 is high as well as unvarying. In our simulated experiments, the estimated

$60 \quad R^{2}$ is lower for the beta-binomial model (0.46) model compared to the

61 binomial (0.63) model, because of its greater SOD. Further, Figure 1c

62 shows how the $\mathrm{R}^{2}$ measure increases from 0.46 to 0.54 , when the slope of

63 the relationship, determined by average detection probability $\mathrm{p}^{*}$, increases

64 from 0.1 to 0.2 in the beta-binomial model.

65

Field sampling concepts

$\underline{\text { Random and representative selection of sites for double-sampling }}$

68 The basic statistical concepts and mathematical formulae applied by

69 Gopalaswamy et al. (2015a,b) assume that the sample size (number of

70 index-calibration data points) is infinite or very large. In practice, this is

71 unrealistic because only a few data points are usually selected for fitting

72 the index-calibration model. But it is essential that the selection of data

73 points should at least representatively retain the same slope and 'flash-

$74 \quad$ light' like SOD shown in Figures $1 \mathrm{a}, 1 \mathrm{~b}$ and 1c. Only with such random site

75 selection can an investigator ensure that model predictions from sampled 
76 points can be extended to generate abundance estimates to larger spatial

77 scales using the double-sampling approach. Therefore, the assumption

78 that the sites sampled do representatively capture the overdispersion

79 pattern in this manner becomes critical to extend inferences about animal

$80 \quad$ abundance to wider areas.

81

(ii) Deconstructing the detection probability $\mathrm{p}^{*}$

83 The detection probability $\mathrm{p}^{*}$ defined here is actually the product of two

84 probabilities: $\alpha$ and $p$, so that $p^{*}=\alpha p$, where $\alpha$ is the proportion of the area

85 within each site that is actually sampled, and $p$ is the probability that an

86 animal within the sampled area in that site is detected during the survey

87 (Williams et al. (2002)). Hence, variation in either $\alpha$ or $p$ will inevitably

88 induce variation in $p^{*}$ (Elliot and Gopalaswamy (2017), Karanth and

$89 \quad$ Nichols (2017)).

90

$91 \quad$ (c) Data analytic concepts

$92 \quad$ Fitting index-calibration models using overdispersed data

93 Ideally these index-calibration models confronting overdispersed data

94 should be defined by likelihood functions for specific data-generating

95 cases (binomial or beta-binomial) (see Richards 2008). However, animal

96 monitoring studies often apply standard linear regression models using

97 ordinary least square (OLS) solutions prescribed in standard textbooks

98 (e.g. Sutherland 2006). There is an inherent problem in doing this, as we

99 illustrate in Figure 3. For $\mathrm{p}^{*}=0.5$, the expected value of $\mathrm{S} \mid \mathrm{N}$ is indicated by

100 a solid dark green line. Let us assume that the average $\mathrm{p}^{*}$ comes from an 
101 underlying beta distribution, with a coefficient of variation $=0.4$. In sign-

102 based indices, $\mathrm{k}$ may be unknown and there is an inherent identifiability

103 issue between $\mathrm{k}$ and $\mathrm{p}^{*}$. So, we assume that the number of sampling

104 occasions $\mathrm{k}$ is fixed and set to 1 (see Gopalaswamy et al. (2015a,b) for the

105 limiting case when $\mathrm{k}=\infty$ ). The circles, distributed around this line,

106 represent one simulated outcome from a set of 20 imaginary data points.

107 When a standard linear regression analysis is conducted on data to relate

108 the variables $\mathrm{S} \mid \mathrm{N}$ and $\mathrm{N}$, an imaginary straight line is constructed (the solid

109 orange line) through the data points. Visually, the placement of this line

110 involves finding an alignment, which will minimize the least distance

111 between each data point and the line. This is called the OLS solution

112 (Casella and Berger 1990). We can use this fitted line to draw inferences

113 about the regression parameters (the slope and intercept) of the linear

114 relationship between $\mathrm{S} \mid \mathrm{N}$ and $\mathrm{N}$. We conducted such a standard linear

115 regression analysis by OLS on our simulated set of data points and plotted

116 the regression line (in solid orange) along with its associated confidence

117 intervals (in dashed orange).

119 We notice here (Figure 2) that while the index $\mathrm{S}$ tracks the variation in $\mathrm{N}$

120 reasonably well, the absolute variation in $\mathrm{S} \mid \mathrm{N}$ itself is quite large,

121 especially when $\mathrm{N}$ increases. For this particular simulation, the OLS

122 solution leads to an overestimation of the slope by a substantial degree

$123 \quad(45.4 \%)$. If this fitted regression line were to be used for making

124 predictions of animal abundance over large scales, seriously biased

125 estimates would result. What is worse is that the direction and magnitude 
126 of the bias depends on the true value of N. Further, we note that the

127 estimated $95 \%$ confidence intervals do not even bind the true expected

128 value (dark green line) in many regions of the graph. Where does this

129 inconsistency come from? The answer is found in statistical theory

130 (Kruskal 1968). It turns out that OLS fits are inefficient in the presence of

131 overdispersion - in our simulated case due to the presence and variation

132 in $\mathrm{p}^{*}$. In fact, this key problem is well recognized in the econometrics

133 literature (Hayashi 2000). This mathematical result means that if

134 overdispersion is present (as it indeed is in most animal survey data sets),

135 abundance predictions employing double sampling approaches using

136 standard linear regression analysis by OLS will not reflect the true

137 underlying uncertainty (eg: poor coverage probability).

139 (ii) How parameter covariance induces artificial non-linearity in index-

$140 \quad$ calibration relationships

141 There is an additional problem affecting the index-calibration method.

142 Often, an inherent covariance can exist between model parameters. The

143 binomial model is denoted as: $E(S \mid N)=k N \alpha p=(k \alpha p) N=\beta N$. Here, we notice

144 that there are three sampling-related parameters, $k, \alpha$ and $p$, and one

145 ecological parameter, N. In practice, if there are underlying ecological or

146 sampling relationships among some of these parameters, the index-

147 calibration relationship will take a non-linear form. In such cases, linear

148 regression models are no longer applicable and their predictions from

149 index calibration data will further be at fault. 


\section{REFERENCES:}

152

Casella, G. \& Berger R. L. (1990) Statistical Inference. Belmont, California: Duxbury Press. estimation of lion density. Cons. Bio, 31, 834-843. experiments: counting tigers at macroecological scales. Methods Ecol Evol. 6: 1055-1066.

Hayashi, F. (2000). Econometrics. Princeton University Press, USA. prey populations. Singapore: Springer. 
Richards, S. A. (2008). Dealing with overdispersed count data in applied ecology. Journal of Applied Ecology: 45, 218-227.

\section{FIGURES}

\section{Figure Legends}

199 Figure 1. Simulation results for hypothetical index-calibration experiments

200 (abundance index $S \mid N$ versus $N$ ) with large sample sizes (2000 data points)

201 are depicted here for the (a) binomial model and (b \& c) beta-binomial 
models. The number of sampling occasions is fixed at $k=3$. We show that the estimated $R^{2}$ value drops from (a) to (b) when a coefficient of variation of 0.4 is applied to the average detection probability parameter $p^{*}$ (set at 0.1 for both cases). Similarly, the $R^{2}$ value increases from (b) to (c) when the average

206 detection probability parameter $p^{*}$ increases from 0.1 to 0.2 . The red lines

207 depict the expected index-calibration relationship, $E(S \mid N)=k N p^{*}$ for the

208 binomial model and $E(S \mid N)=k N[a /(a+b)]$ for the beta-binomial model. The

209 parameters $a$ and $b$ are estimated from the given coefficient of variation by the 210 formulae provided in Gopalaswamy et al. (2015a,b).

212 Figure 2. Illustration of the extent of dispersion under the beta-binomial model

213 of index-calibration, $N$ versus $S$ (which is conditional on $N$ ). The

214 overdispersion is caused by the presence of a variable detection probability, 215 so that $S \mid N \sim \operatorname{Beta-binomial}(k N, a, b)$ with the corresponding $a$ and $b$ set to 216 reflect a coefficient of variation of 0.4 around the average $p^{*}$. The sampling 217 occasion $k$ is assumed to be a constant with a value of one. The dark green 218 circles represent a random selection of data points from the specified

219 distribution, where mean $=\mathrm{Na} /(\mathrm{a}+b)$ and variance

$220=[N a b(a+b+N)] /[(a+b) 2(a+b+1)]$, for average $\mathrm{p}^{*}=0.5$ 


\section{$244 \quad$ Figures}

245

246 Figure 1a)

247

248 
Binomial model of index counts $\left(k=3, p^{*}=0.1\right)$

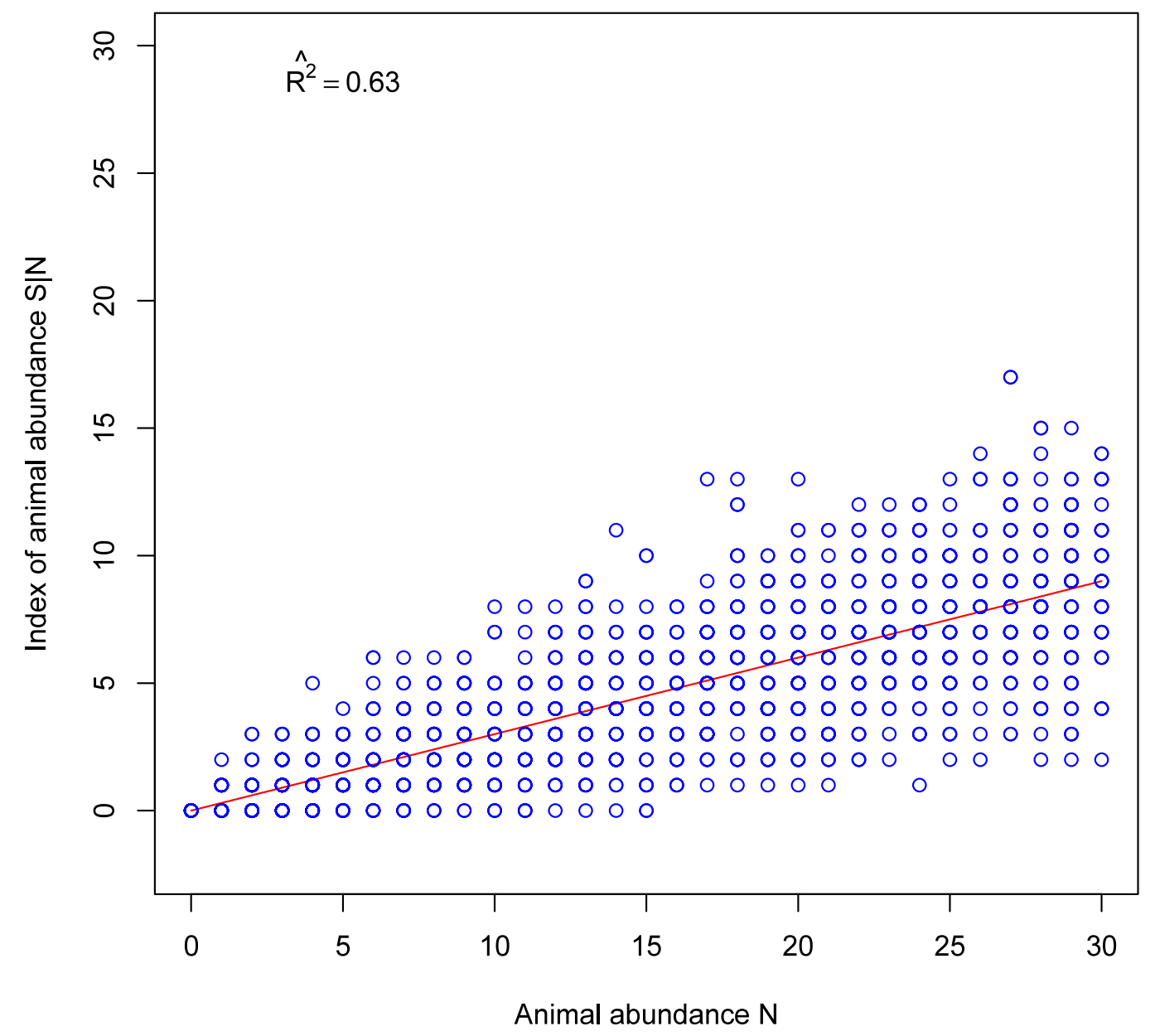


Beta-binomial model of index counts $\left(k=3\right.$, Mean $\left.p^{*}=0.1\right)$

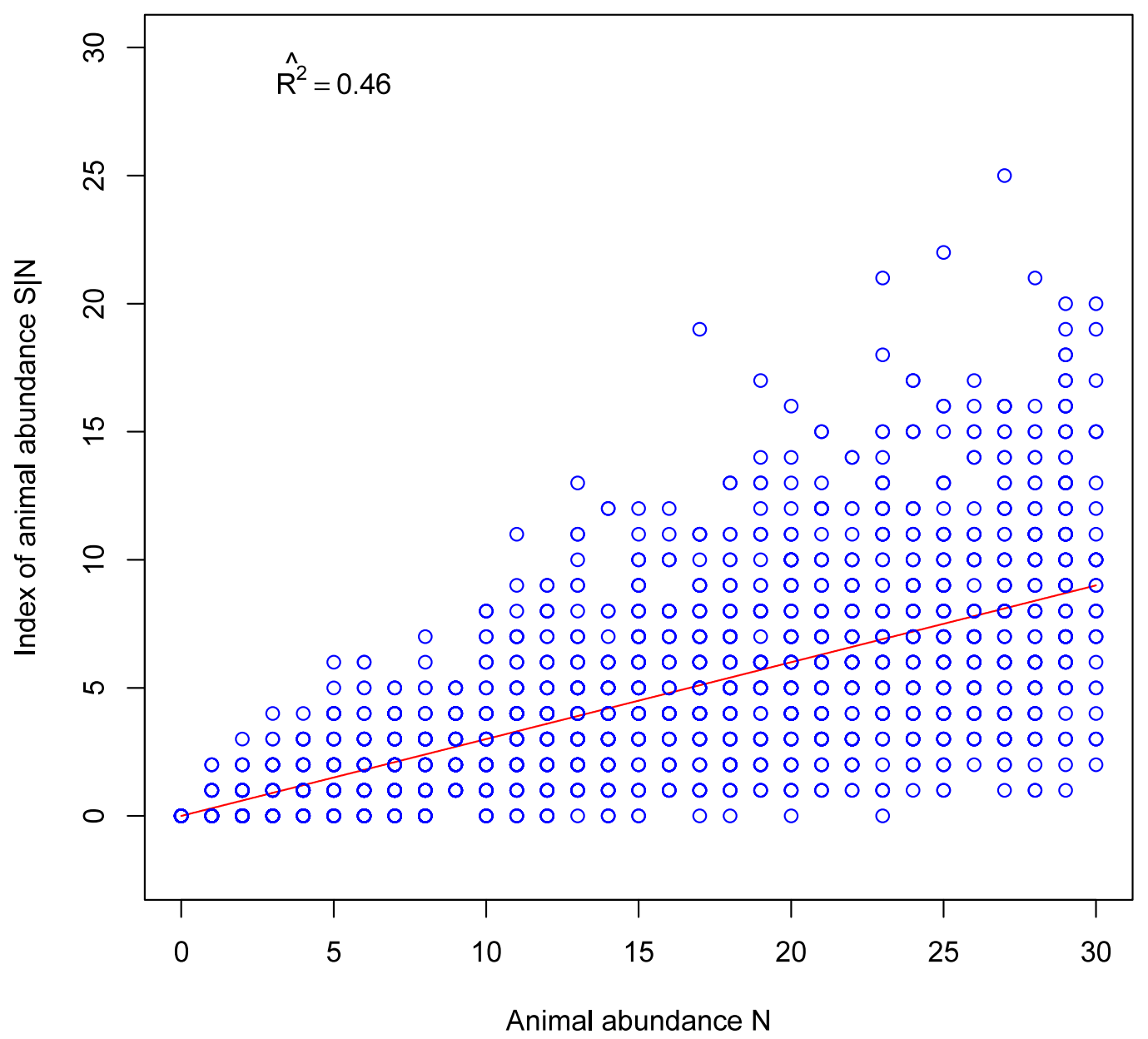


Beta-binomial model of index counts $\left(k=3\right.$, Mean $\left.p^{*}=0.2\right)$

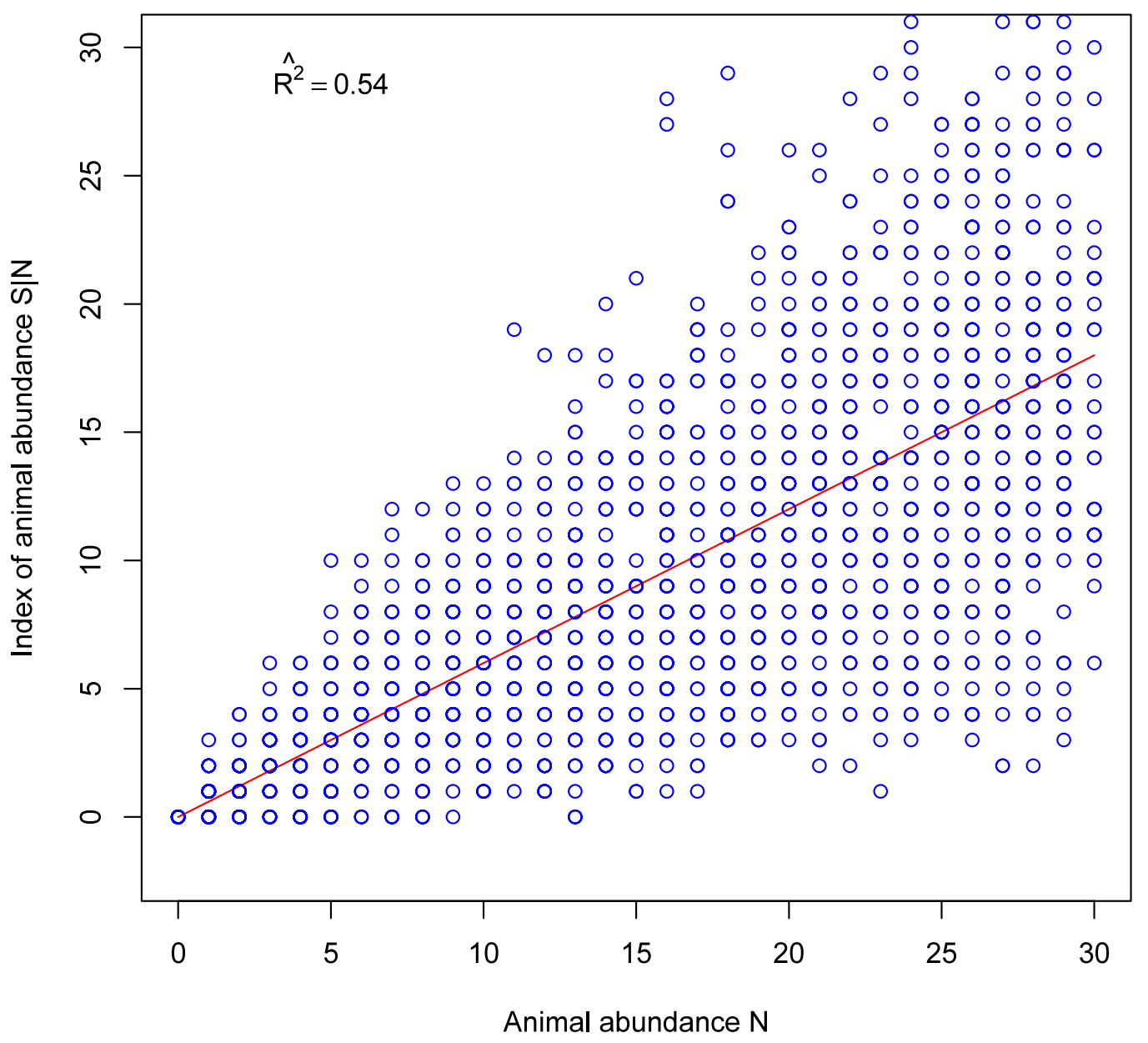




\section{Overdispersion in beta-binomial model}

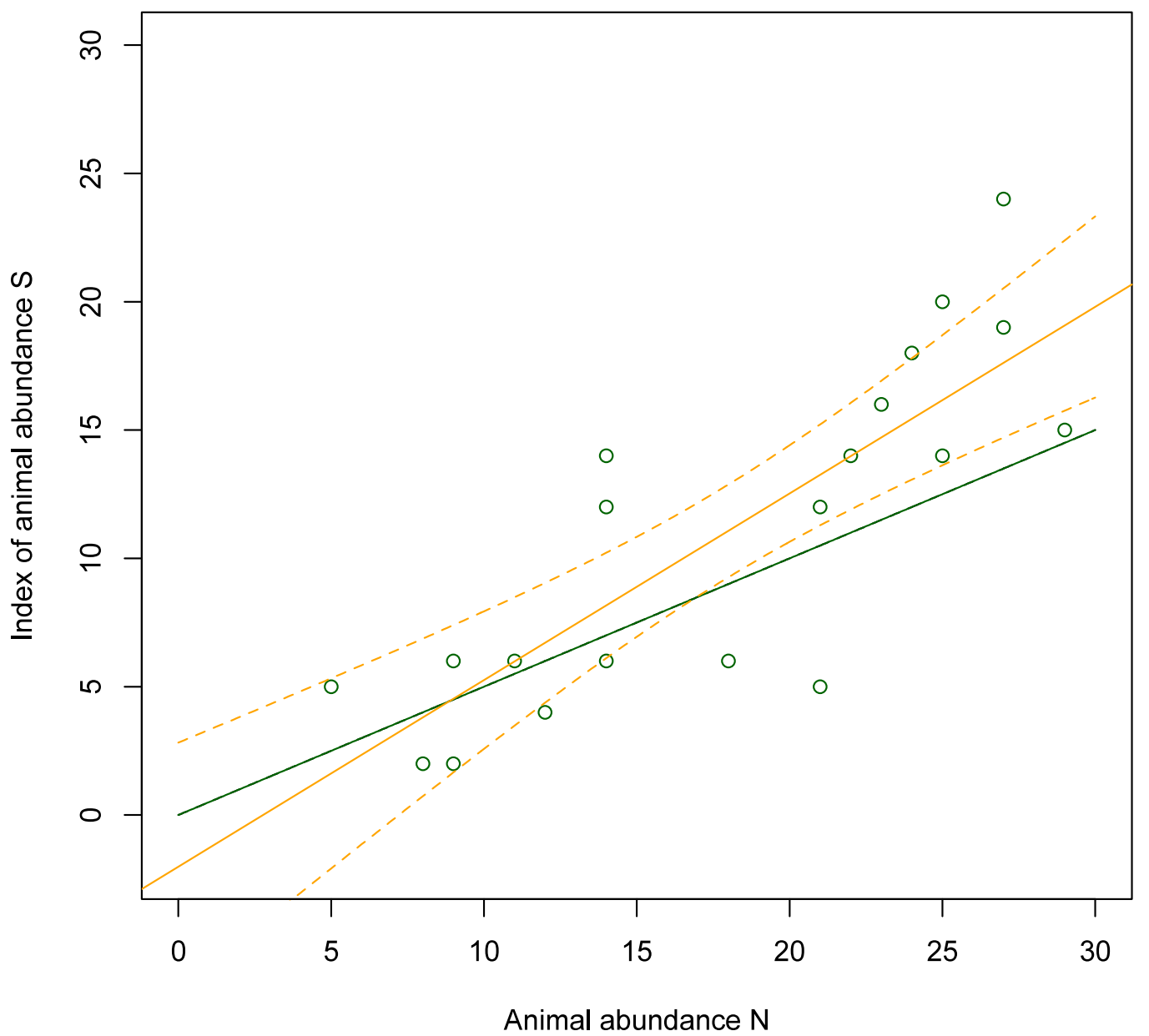

